\title{
Gilles Bertrand et Pierre Serna (dir.), La République
} en voyage, $1770-1830$

Rennes, Presses Universitaires de Rennes, 2013

\section{Annie Duprat}

\section{(2) OpenEdition}

\section{Journals}

Édition électronique

URL : https://journals.openedition.org/ahrf/13308

DOI : 10.4000/ahrf.13308

ISSN : 1952-403X

Éditeur :

Armand Colin, Société des études robespierristes

Édition imprimée

Date de publication : 15 octobre 2014

Pagination : 204-204

ISBN : 978-2-20-092927-5

ISSN : 0003-4436

Référence électronique

Annie Duprat, « Gilles Bertrand et Pierre Serna (dir.), La République en voyage, 1770-1830 ». Annales

historiques de la Révolution française [En ligne], 377 | juillet-septembre 2014, mis en ligne le 15

septembre 2014, consulté le 01 juillet 2021. URL : http://journals.openedition.org/ahrf/13308 ; DOI https://doi.org/10.4000/ahrf.13308

Ce document a été généré automatiquement le 1 juillet 2021.

Tous droits réservés 


\section{Gilles Bertrand et Pierre Serna (dir.), La République en voyage, 1770-1830}

Rennes, Presses Universitaires de Rennes, 2013

\section{Annie Duprat}

\section{RÉFÉRENCE}

Gilles Bertrand et Pierre Serna (dir.), La République en voyage, 1770-1830, Rennes, Presses Universitaires de Rennes, 2013, 442 p., ISBN 978-2-7535-2805-5, $24 €$.

1 Rendre compte des actes d'un colloque est un exercice routinier autant que périlleux : routinier parce que les colloques sont nombreux, périlleux parce qu'il s'agit d'une succession d'articles portant sur un sujet souvent assez large pour ouvrir la voie à des excursus pas toujours heureux. Périlleux aussi en raison de l'inégale qualité desdits articles. Alors, que doit faire le recenseur : décrire le livre de façon neutre, en essayant de «n'oublier personne »? Focaliser son attention sur les pages qui lui paraissent les plus congruentes avec le projet initial? S'en tenir aux intentions des organisateurs telles qu'exprimées dans l'introduction? Même si j'ai veillé à l'équanimité du commentaire, que, par avance, les contributeurs me pardonnent s'ils constatent des inégalités dans le traitement réservé à chacun d'eux dans la présente recension.

2 Le titre, La République en voyage, ainsi que le cadre chronologique (une période révolutionnaire au sens large) appelle à picorer, à flâner parmi tant d'articles venus de tant de pays différents. Vingt-six contributions sont organisées en cinq parties :

1. La mise en place d'un voyage républicain

2. Révolution, conflits et voyages mouvementés

3. La république traduite, la république en transfert

4. Le voyage. Fabrique des savoirs républicains 
5. Au bout du voyage. Héritage d'une génération.

3 Le projet de la rencontre scientifique qui a donné lieu à cette publication était de montrer comment le voyage (ou les voyages) des protagonistes a (ont) forgé les consciences individuelles des voyageurs et comment, en retour, ils ont diffusé les expériences révolutionnaires de la période. Le "Grand Tour", pratique éducative lancée par et pour les nobles anglais à la fin $\mathrm{du} \mathrm{xvI} \mathrm{e}^{\mathrm{e}}$ siècle confine au voyage touristique à la fin $d u \mathrm{XVIII}^{\mathrm{e}}$. Les témoignages sont nombreux et créent une sorte d'espace virtuel des émotions. Car les expériences d'échanges et les comparaisons se font, à l'intérieur de l'Europe mais aussi avec les Amériques en particulier. Vaste ambition qui explique l'aspect mosaïque mais aussi la richesse du recueil. Gilles Bertrand et Pierre Serna ont rédigé une introduction très détaillée et nourrie de références. L'index des noms de personnes figurant en fin d'ouvrage est particulièrement indispensable pour le lecteur moins frotté à l'histoire des voyages; un index des lieux, avec croisement avec les destinations, aurait été bienvenu. En effet, si le voyage en Suisse d'Helen Maria Williams est assez connu, grâce à sa traduction par Jean-Baptiste Say, celui de Bourgoing en Espagne (1788) ou les périples en Italie de divers voyageurs, mentionnés note 37 p. 21, par exemple, le sont beaucoup moins. Les voyageurs dont les récits sont présentés ici sont des passionnés de découvertes et, souvent, des militants. C'est ce qui donne tout le prix à leurs témoignages. Au « voyage républicain » pourtant va s'ajouter «l'anti-voyage républicain » à partir de 1792.

4 Au fil de la première partie, nous suivons plusieurs voyageurs français à travers l'Europe (Hollande, Suisse, mais aussi les îles britanniques) étudiés par Annie Jourdan. Elle conclut à la vitalité d'un républicanisme pré-révolutionnaire, des années avant l'établissement de la Constitution américaine. Le regard porté par le polonais Félix Oraczewski sur la France pré-révolutionnaire d'abord, puis en 1791-1792, montre une grande vigilance et une perspicacité certaine qui lui permettait de distinguer les failles derrière le vernis (Alexandra Janiszewska). Le Hollandais Lambert Van Eck voyage en 1788 (étude par Arianne Bagermann et Rudolf Dekker). Il raconte les tracasseries aux différents péages, les discussions sur les mouvements révolutionnaires qui se multiplient à travers l'Europe. Les échanges entre les exilés et les voyageurs tiennent à la fois du tourisme moderne et de la construction des réseaux militants. "Les émigrés genevois de 1782 sont, avec les loyalistes américains, parmi les premiers exilés produits par les bouleversements révolutionnaires de la fin du XvIII ${ }^{e}$ siècle, avant les émigrés français ou les exilés italiens de la Révolution française » écrit Rahul Markovits dans une brève étude portant sur les exilés genevois. On voit comment plusieurs d'entre eux ont cru pouvoir opérer une "transplantation» en Angleterre, avant de revenir progressivement à Genève ou de s'installer dans les Provinces-Unies.

5 La seconde partie du livre s'attache plutôt aux aspects sombres, les voyageurs se trouvant en butte aux violences et aux menées contre-révolutionnaires. Tel est le sort de ces Français suspectés de jacobinisme en Bohême et en Moravie entre 1790 et 1800 présentés par Daniela Tinkovà. Pourchassés par les polices locales, ils ont de ce fait rempli les archives des services de surveillance. Davantage que des révolutionnaires militants, ce sont des Français fuyant la disparition de la monarchie dans leur pays. Sont-ils pour autant des émigrés au sens traditionnellement accepté ? Pas tous, car il y a des déserteurs de l'armée républicaine par exemple. On touche ici aux limites des définitions des comportements particuliers durant un événement collectif d'une telle ampleur. Nicole Pellegrin est aussi confrontée à cette question de la définition 
lorsqu'elle observe plusieurs religieuses-voyageuses entre 1789 et 1815. Par l'expérience inédite de l'exil, ces femmes cherchent-elles le martyre ? L'exil intérieur, celui des girondins, serait une autre expérience d'exil, selon Guillaume Mazeau. Contraints à la clandestinité, les députés girondins découvrent la France dans ses profondeurs et font, à la suite du Louis XVI de retour de Varennes, l'amer constat qu'il n'existe pas de "bon pays ", ni pour eux-mêmes ni pour quiconque. Si le terme n'était pas anachronique, on pourrait écrire qu'ils font le constat de la rugosité du réel en descendant des tribunes des clubs, sociétés ou assemblées! Pierre Claude Gerlain, originaire de Melun, est un ardent républicain installé aux Antilles des années avant la Révolution. Il tient un journal durant plusieurs mois en 1794, riche document - car la Guadeloupe tombe sous la domination anglaise en avril - exploité par Frédéric Régent. Ayant élaboré des tableaux statistiques à partir des données de Gerlain, l'auteur nous montre comment " être Républicain sous les Tropiques », face à la Royal Navy.

6 La troisième partie déplace la question du voyage des personnes, le voyage physique en quelque sorte, vers celui des idées, de la "République des Lettres». Il faut alors reprendre les textes et leurs exégèses successives. Lucia Bergamasco revient sur les écrits de l'Américain James Madison tirés de ses lectures de textes juridiques antiques et modernes et de sa correspondance. Ainsi s'éclairent mieux les choix du quatrième président des États-Unis. Anne Simonin revient sur l'élaboration de la Loi nouvelle, depuis les diverses coutumes d'Ancien Régime jusqu'à 1792 et sa traduction/ acceptation/mise à distance dans les faits. Élaborée en France, la Loi révolutionnaire peut alors voyager pour diffuser ses idéaux dans les pays limitrophes. "Les voyages forment la jeunesse », dit-on couramment, paraphrasant Montaigne. Gabor Gelléri en fait une belle illustration en isolant un texte contenu dans la collection Portiez de l'Oise, Des voyages. De leur utilité dans l'éducation, le député de l'Oise ayant souvent confié ses doutes quant à la régénération de l'Homme, fût-il devenu citoyen! Dans un monde qui se rétrécit sans cesse au gré des multiples voyages des uns et des autres, les nécessités de la traduction se font jour. Jean-Luc Chappey étudie la façon dont Griffet de Labaume l'a envisagée comme une pratique politique. Après une brève reprise de la biographie d'un de ces acteurs du Cercle social, pensionné par la Convention, puis l'une des «petites mains » de François de Neufchâteau au ministère de l'Intérieur en 1798, Chappey montre que Labaume a toujours présente à l'esprit la nécessité de faire connaître informations et opinions au plus grand nombre, par-delà les frontières linguistiques.

7 Tout naturellement, nous parvenons à la partie suivante qui s'occupe de la « fabrique des savoirs républicains ». Nous y ferons plus ample connaissance avec Jean-François Bourgoing, auteur du Tableau de l'Espagne moderne, étudié par Jean-René Aymes. Le livre, réédité à plusieurs reprises entre 1788 et1797, brosse des portraits à multiples facettes de l'Espagne et des Espagnols, déconstruisant un certain nombre de stéréotypes (paresse, orgueil et vanité par exemple). Quant à Helen Maria Williams, cette Anglaise dont le voyage en Suisse a trouvé en la personne de Jean-Baptiste Say un traducteur prestigieux en 1798; elle est ici étudiée par Bernard Gainot qui montre sa prise de distance avec l'idéal-type du « citoyen de Genève ». Historien des sciences, Pierre-Yves Lacour fait un tableau détaillé des missions scientifiques en Italie du Nord entre 1794 et 1797 en insistant sur les réactions (ou plutôt, en l'occurrence, l'absence de réactions) face aux confiscations destinées à enrichir les collections des musées. Ces voyages ne sont pas des razzias mais sont l'occasion pour les savants de rencontrer leurs confrères et d'échanger avec eux. Retour aux Provinces-Unies avec l'article de Madeleine Van 
Strien-Chardonneau sur le voyage en 1794-1795 du commissaire de la République André Thouin. Au-delà de la botanique, il y examine l'économie (les industries) mais aussi l'assistance aux pauvres. Sa description de l'établissement de charité d'Amsterdam est un modèle du genre. Jean Dhombres, sous un titre énigmatique, «Les atours de la science républicaine en voyage à la toute fin du XvIII ${ }^{\mathrm{e}}$ siècle et les effets du retour » cherche à montrer comment la pompe des voyages - en présence de Bonaparte parfois - a permis de créer une sociabilité républicaine parmi les savants mais aussi parmi les politiques. Il est à noter que les nombreuses illustrations données à l'appui de la démonstration ont pâti d'une très mauvaise qualité de reproduction.

Le titre de la dernière partie, «Au bout du voyage. L'héritage d'une génération » est assez englobant. On y trouve une série d'études de cas. Sylvie Kleinman revient sur le cas de Wolfe Tone, révolutionnaire irlandais qui voyage dans la France du Directoire ; il décrit par le menu son voyage, ses rencontres, ses enthousiasmes et ses étonnements. Anna Maria Rao étudie le voyage en Catalogne du capitaine napolitain Gabriele Pepe en 1807-1808. En quelques pages sont brossées les principales caractéristiques du texte de Pepe qui a observé le sud de la France et ses habitants avant de gagner la Catalogne. La Fayette en Amérique ne pouvait pas être absent de ce volume. Élisabeth Liris reprend les écrits du "Héros des deux-mondes " pour y trouver des réflexions nouvelles sur le républicanisme ou la démocratie royale. L'originalité de l'article réside dans l'entrecroisement des textes de La Fayette avec le traitement qu'en ont fait les historiens ultérieurs. En contrepoint, le Barère de Maïté Bouissy est un «voyageur immobile ", du moins c'est le titre donné à la contribution. Car, entre ses prisons, ses voyages à l'intérieur de la France et la richesse de ses écrits, le conventionnel est loin d'être immobile. Les textes des exilés italiens comme Ugo Foscolo, qui ont voyagé à travers l'Europe à partir de 1821 sont étudiés par Olaf Müller. Il montre que ces précurseurs de la révolte composent en quelque sorte un Panthéon pour le Risorgimento.

9 La conclusion de Pierre Serna démontre que la République, qui ne cesse d'être recherchée, ne cesse pas non plus de s'éloigner. Objet de civilisation, elle est nourrie par les savoirs venus de partout et enrichie par les voyages. Pourtant, ce qu'apportent justement la modernité et les voyages, c'est la diffusion de la République, même si son triomphe réel n'est jamais assuré. 